\title{
The magnitude of weight loss induced by metformin is independently associated with BMI at baseline in newly diagnosed type 2 diabetes: Post-hoc analysis from data of a phase IV open-labeled trial
}

\author{
Lingli Zhou ${ }^{\mathrm{B}-\mathrm{F}}$, Xiaoling Cai ${ }^{\mathrm{B}-\mathrm{C}, \mathrm{F}}$, Wenjia Yang ${ }^{\mathrm{B}-\mathrm{C}, \mathrm{F}}, \mathrm{Xueyao} \mathrm{Han}^{\mathrm{A}, \mathrm{E}-\mathrm{F}}$, Linong Ji, ${ }^{\mathrm{A}-\mathrm{F}}$ \\ Department of Endocrinology and Metabolism, Peking University People's Hospital, Beijing, China \\ A - research concept and design; $\mathrm{B}$ - collection and/or assembly of data; $\mathrm{C}$ - data analysis and interpretation; \\ $D$ - writing the article; $E$ - critical revision of the article; $F$ - final approval of article
}

\section{Address for correspondence \\ Linong Ji}

E-mail:prof_jilinong@aliyun.com

Funding sources

This study was funded by Merck Serono Co. Ltd. China, an affiliate of Merck KGaA, Darmstadt,

Germany and the National Key Basic Research

Program of China (973 Program 2011CB504000).

\section{Conflict of interest}

None declared

\section{Acknowledgements}

The authors thank dr Yingying Luo from the Department of Endocrinology and Metabolism, Peking University People's Hospital for excellent advice on the statistical analysis.

Received on December 03, 2015

Revised on January 13, 2016

Accepted on May 05, 2016

\begin{abstract}
Background. The impact of metformin on body weight is variable in T2DM patients among studies. The reasons for the discrepancies are still unknown.

Objectives. We aimed to find out the possible predictive factors of weight change induced by metformin based on an analysis of a phase IV open-labeled trial.

Material and methods. We performed a sub-analysis from a prospective, multi-center phase IV open-labeled study in which 371 Chinese newly-diagnosed type 2 diabetes patients received 16 weeks' extended-release metformin monotherapy. The clinical characteristics including weight and laboratory assessments of subjects were collected every 4 weeks. The weight changes from baseline to week 4, 8, 12 and 16 were calculated respectively. The patients were divided into 4 groups by quartile statistics method according to magnitudes of weight change.
\end{abstract}

Results. Of 371 enrolled patients, 324 patients had the weight records from baseline to week 16. The weight decreased gradually with each visit $(p<0.001)$ and the average weight loss was $2 \mathrm{~kg}$ after 16 weeks' treatment of metformin. The patients with higher BMl and bigger waist circumference at baseline showed a more pronounced weight loss. However, the magnitudes of weight loss were independently associated with BMI at baseline only.

Conclusions. Metformin can lead to weight loss gradually in newly-diagnosed type 2 diabetes patients. The magnitude of weight loss was independently and only associated with baseline BMI.

Key words: obesity, weight loss, metformin, Asia diabetes

DOI

10.17219/acem/63025

Copyright

Copyright by Author(s)

This is an article distributed under the terms of the

Creative Commons Attribution Non-Commercial License

(http://creativecommons.org/licenses/by-nc-nd/4.0/) 
Lines of evidence have shown that metformin not only consistently lowers blood glucose, but also reduces the risk of cardiovascular diseases, micro-vascular diseases and cancer. ${ }^{1-3}$ Compared to sulfonylureas, metformin seldom causes weight gain and hypoglycemic attacks. ${ }^{4}$ As a result, metformin is recommended as the first-line glucose-lowering medication by almost all international guidelines. ${ }^{5}$ In China, recent evidence shows that the glycemic response to metformin monotherapy is similar among normal weight, overweight and obese patients. ${ }^{6}$ Consistently, the guidelines of the Chinese Diabetes Society (CDS) in 2010 also recommended metformin as the first-line drug in patients with newly-diagnosed type 2 diabetes regardless of BMI.?

However, a national survey conducted in China showed that only $52 \%$ of prescriptions were in line with the guidelines. ${ }^{7}$ One of the important reasons is that some prescribing doctors worry about the well-known "side-effect" of metformin (weight reduction), since Asians have lower BMI than Caucasians and the cut-off of BMI for defining obesity is different among regions. Patients with "normal weight" in Asia may be defined as lean patients in Europe and America, who were seldom investigated in connection withthe effects of metformin. Weight loss, the beneficial "side-effect" of metformin on patients excessive weight loss with "normal weight" in Europe and America, may cause in Asian patients with "normal weight". Therefore, it is necessary to investigate the effects of metformin on weight in Chinese patients with type 2 diabetes with normal weight and overweight.

Our study aimed to analyze the effect of metformin on body weight change and to investigate the risk factors associated with the magnitude of metformin-induced weight loss in newly-diagnosed Chinese type 2 diabetes.

\section{Material and methods}

\section{Design and participants}

We analyzed the data from a prospective, multi-center, phase IV, open-label study carried out in 371 Chinese patients aged 23-77 years who were newly diagnosed with type 2 diabetes. This study was registered at ClinicalTrials.gov, NCT 00778622 and conducted at 20 sites in China between November 19, 2009 and March 15, 2011. The protocol was approved by the Ethics Committee from each clinical site. Metformin (metformin XR [Glucophage ${ }^{\circledR}$ XR], Bristol Myers Squibb) was administered at an initial dose of $500 \mathrm{mg} /$ day taken orally with the evening meal. After 1 week, dosage was titrated upwards in increments of $500 \mathrm{mg}$ weekly until the maximum daily dose of $2,000 \mathrm{mg} /$ day was reached, unless intolerance or hypoglycemia was experienced. From week 4, the maximum daily dose was 2,000 mg/day if FPG was > $7.0 \mathrm{mmol} / \mathrm{L}$ (126 mg/dL).

\section{Statistical analyses}

The data was analyzed using SPSS 16.0 (IBM, Inc, New York, USA). Continuous variables were expressed as mean \pm SD. Since TG did not follow a normal distribution, the values were log transformed and displayed as averages and upper and lower quartiles. The changes of weight loss with time were analyzed using the mixed effect model. The differences of continuous parameters between the 2 groups were evaluated by t-test. The differences of proportions between the 2 groups were analyzed by $\chi^{2}$ test. The differences of continuous parameters that had normal distribution among the groups were analyzed by ANOVA test. Pearson's correlation analysis was carried out to analyze the relationship between 2 variables that meets normal distribution, while Spearman correlation analysis was used for those variables that didn't meet normal distribution. A stepwise linear regression analysis was performed to determine the independent factors associated with weight loss.

\section{Results}

\section{Effect of 16-week metformin monotherapy on weight of patients with newly- diagnosed type 2 diabetes}

The mean weight of the subjects at baseline was $72.4 \pm 13.1 \mathrm{~kg}, 71.2 \pm 12.6 \mathrm{~kg}$ at week $4,70.5 \pm 13.3 \mathrm{~kg}$ at week $8,70.2 \pm 12.7 \mathrm{~kg}$ at week 12 and $70.0 \pm 12.7 \mathrm{~kg}$ at week 16, respectively. The mixed effect model test showed that weight decreased with time (Fig. 1A, p < 0.001).

According to recommendations by the Working Group on Obesity in China, $18.5 \mathrm{~kg} / \mathrm{m}^{2} \leq \mathrm{BMI} \leq 23.9 \mathrm{~kg} / \mathrm{m}^{2}$ is defined as normal weight, and $>24 \mathrm{~kg} / \mathrm{m}^{2}$ and $>28 \mathrm{~kg} / \mathrm{m}^{2}$ are defined as overweight and obese, respectively. ${ }^{8}$ We found that after metformin treatment, the proportion of obese patients decreased $(\mathrm{p}=0.005)$. The proportion of overweight patients with also decreased, whereas the proportion of patients with normal weight increased, although not significantly (Fig. 1B). Only three subjects fill into the "lean" group after treatment, since they had had a relatively low BMI at baseline $\left(18.6 \mathrm{~kg} / \mathrm{m}^{2}, 19.3 \mathrm{~kg} / \mathrm{m}^{2}\right.$ and $18.5 \mathrm{~kg} / \mathrm{m}^{2}$, respectively). Their BMI were $18.2 \mathrm{~kg} / \mathrm{m}^{2}$, $18.1 \mathrm{~kg} / \mathrm{m}^{2}$ and $18.3 \mathrm{~kg} / \mathrm{m}^{2}$, respectively, at week 12 and unchanged at week 16 .

\section{Characteristics of patients with different response of weight change to metformin treatment}

To explore the baseline characteristics of patients with different response of weight change to metformin treatment, we divided them into 4 groups by the quartile method based on the magnitude of weight loss at week 16 : 
Fig. 1. The weight change over time after metformin treatment. A) The weight change trend by visit (mix effect model). B) proportion of patients in 4 groups based on BMI. "Lean" was defined as BMI $<18.5 \mathrm{~kg} / \mathrm{m}^{2}$, "Normal" was defined as $18.5 \mathrm{~kg} / \mathrm{m}^{2}<\mathrm{BMI}<\mathrm{BMI} 28 \mathrm{~kg} / \mathrm{m}^{2}$; ${ }^{*}$ represents p $<0.05</$ BMI
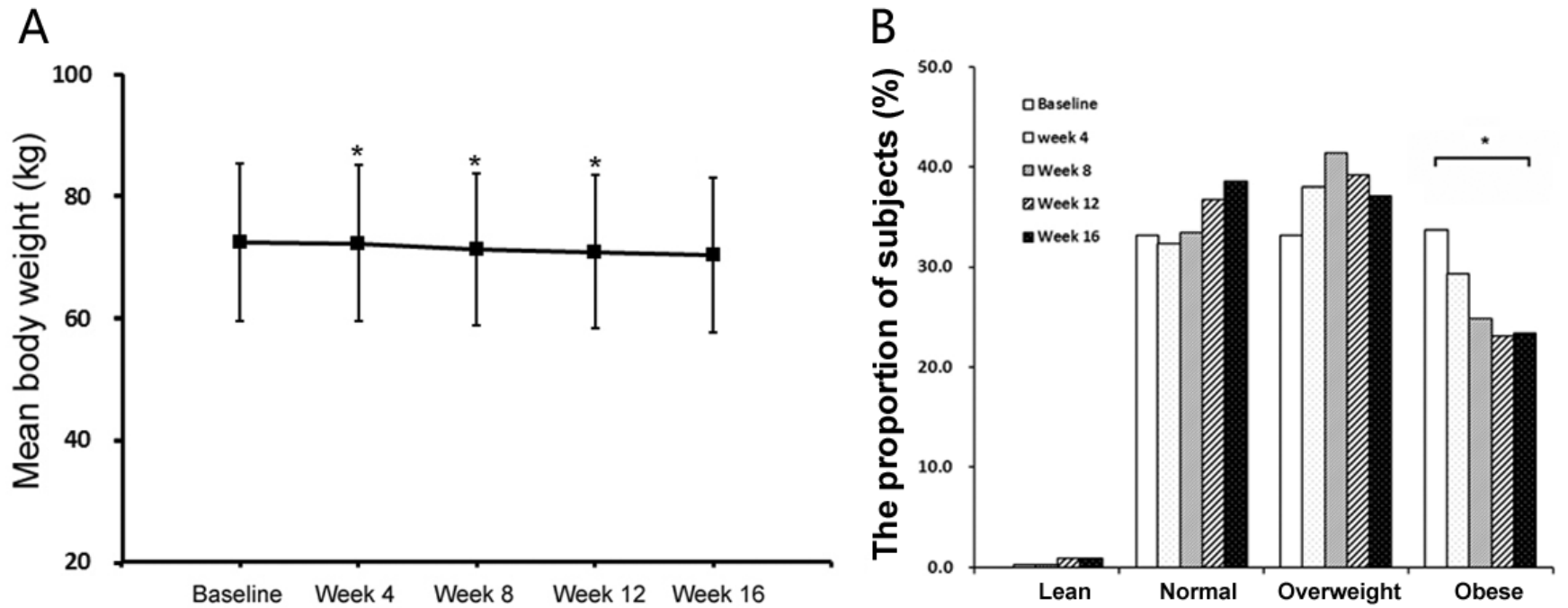

Fig. 2. Correlation of weight (A), serum albumin level (B), BMI (C) and waistline (D) at baseline with the magnitude of weight loss Pearson's correlation coefficient $(r)$ is shown
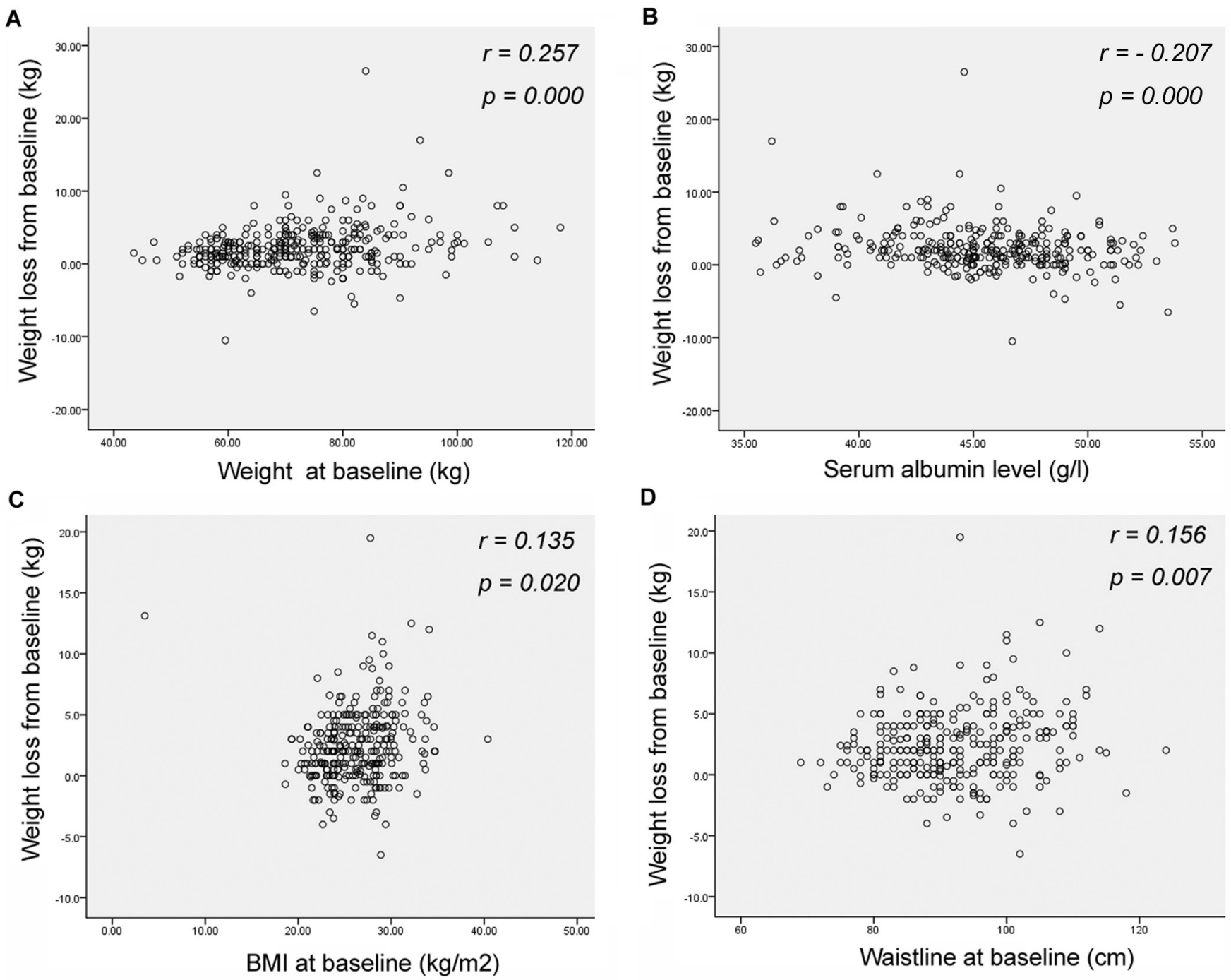

D

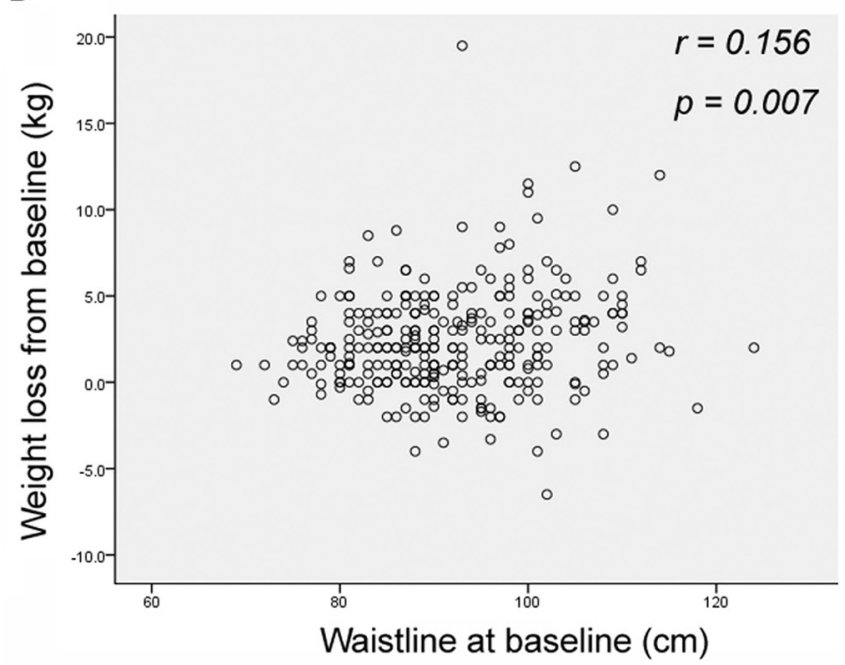


Table 1. Clinical characteristics of patients in the 4 groups

\begin{tabular}{|c|c|c|c|}
\hline & $\begin{array}{l}\text { Group } 1(n=84) \\
W L<=0.5 \mathrm{~kg}\end{array}$ & $\begin{array}{c}\text { Group } 2(\mathrm{n}=91) \\
0.5 \mathrm{~kg}<\mathrm{WL}<=2.0 \mathrm{~kg}\end{array}$ & $\begin{array}{c}\text { Group } 3(n=79) \\
2.0 \mathrm{~kg}<\mathrm{WL}<=4.0 \mathrm{~kg}\end{array}$ \\
\hline Age (years) & $50.57 \pm 10.57$ & $53.71 \pm 10.44$ & $53.97 \pm 10.27$ \\
\hline BMI $\left(\mathrm{kg} / \mathrm{m}^{2}\right)$ & $25.52 \pm 3.27$ & $25.71 \pm 3.49$ & $26.23 \pm 3.82$ \\
\hline Waistline $(\mathrm{cm})$ & $90.89 \pm 8.58$ & $90.58 \pm 10.51$ & $92.77 \pm 9.37$ \\
\hline $\mathrm{SBP}(\mathrm{mm} \mathrm{Hg})$ & $125.81 \pm 15.13$ & $130.93 \pm 15.97$ & $128.48 \pm 15.86$ \\
\hline $\mathrm{DBP}(\mathrm{mm} \mathrm{Hg})$ & $79.80 \pm 9.22$ & $81.00 \pm 8.60$ & $79.94 \pm 10.12$ \\
\hline Weight (kg) & $70.11 \pm 12.02$ & $70.08 \pm 13.91$ & $71.74 \pm 12.46$ \\
\hline $\mathrm{HIP}(\mathrm{cm})$ & $97.38 \pm 8.19$ & $98.52 \pm 9.07$ & $97.53 \pm 8.52$ \\
\hline GHBA1C (\%) & $8.43 \pm 0.82$ & $8.48 \pm 0.80$ & $8.38 \pm 0.80$ \\
\hline FBG (mmol/L) & $8.71 \pm 2.02$ & $8.71 \pm 2.06$ & $8.65 \pm 2.04$ \\
\hline UA (mmol/L) & $300.63 \pm 107.72$ & $292.10 \pm 132.38$ & $279.16 \pm 132.57$ \\
\hline $\operatorname{ALT}(U / L)$ & $29.60 \pm 14.29$ & $28.41 \pm 13.52$ & $28.39 \pm 15.45$ \\
\hline CRE (umol/L) & $65.58 \pm 14.60$ & $72.33 \pm 18.38$ & $68.30 \pm 19.37$ \\
\hline Cumulative dosages (mg) & $169708.3 \pm 18886.50$ & $173505.5 \pm 17930.81$ & $171158.2 \pm 18543.70$ \\
\hline LDL (mmol/L) & $3.20 \pm 0.87$ & $3.17 \pm 0.82$ & $2.98 \pm 0.87$ \\
\hline LgTG (mmol/L) & $0.60 \pm 0.55$ & $0.58 \pm 0.60$ & $0.55 \pm 0.67$ \\
\hline $\mathrm{HDL}(\mathrm{mmol} / \mathrm{L})$ & $1.13 \pm 0.24$ & $1.13 \pm 0.25$ & $1.14 \pm 0.27$ \\
\hline AE of gastrointestinal & 14.26 & 15.45 & 13.41 \\
\hline GHBA1c reduction (\%) & $1.78 \pm 0.84$ & $1.92 \pm 0.90$ & $1.81 \pm 0.96$ \\
\hline FBG reduction (mmol/L) & $1.86 \pm 2.10$ & $2.07 \pm 1.92$ & $2.08 \pm 1.92$ \\
\hline
\end{tabular}

group 1 ( $\mathrm{n}=84)$, in which the subjects had a weight loss $\leq 0.5 \mathrm{~kg}$, group $2(\mathrm{n}=91)$, in which subjects had $0.5 \mathrm{~kg}$ $<$ weight loss $\leq 2.0 \mathrm{~kg}$, group $3(\mathrm{n}=79)$ in which subjects had a $2.0 \mathrm{~kg}<$ weight loss $\leq 4.0 \mathrm{~kg}$, and group $4(\mathrm{n}=70)$ in which subjects had a weight loss $>4 \mathrm{~kg}$ (Table 1$)$. There was no difference in age, systolic pressure, diastolic pressure, prevalence of hypertension, TCHOL, HDL-C, TG, LDL-C, fasting glucose and HBA1c. Compared to the subjects with the lowest quartile, the patients with the highest quartile magnitude of weight loss had the heaviest weight ( $p=0.0001)$, highest BMI ( $p=0.0002)$, largest waistline $(\mathrm{p}=0.016)$ and lowest level of serum albumin $(\mathrm{p}=0.013)$ at baseline.
We also compared the changes of metabolic parameters, drug dosages and gastrointestinal intolerances among the 4 groups. There were no differences among the groups regarding the improvement of glucose control, as neither the change of HbA1c (plasma glucose concentration) nor FBG (fasting blood glucose) showed differences among the four groups. There were also no differences among the groups of drug administered dosages $(\mathrm{p}=0.4603)$ and gastrointestinal intolerances $(\mathrm{p}=0.0645)$.

The correlations of the clinical parameters to the magnitude of weight loss are shown in Table 2 and Fig. 2. Weight loss was correlated with weight $(r=0.257$, $\mathrm{p}=0.000)$, BMI $(\mathrm{r}=0.135, \mathrm{p}=0.020)$ and waistline 
$(\mathrm{r}=0.156, \mathrm{p}=0.007)$ at baseline, whereas inversely associated with the serum albumin $(r=-0.207, \mathrm{p}=0.000)$ at baseline.

\section{Predictors for weight loss induced by metformin treatment}

We then performed a stepwise linear regression analysis to identify predictors for magnitude of weight loss after 16-week metformin treatment using independent variables including age, HbA1c, fasting glucose, lipid measurements, systolic pressure, diastolic pressure, serum albumin at baseline, the incident rate of gastrointestinal AE (adverse events) and the cumulative dosages of metformin. The analysis revealed that only baseline BMI was an independent factor contributing to magnitude of weight loss (Table 3).

\section{Discussion}

In this post-hoc analysis, we focused on the clinical characteristics associated with weight loss during metformin treatment. Our results showed that 16 -week metformin treatment could induce a mean $2.4 \mathrm{~kg}$ weight loss. The magnitude of weight loss is positively associated with the baseline weight. Interestingly, it is inversely associated with the serum albumin level at baseline and the incident rate of gastrointestinal AE. To our knowledge, few studies have investigated the predictors of weight loss induced by metformin. Moreover, this is the first report that serum albumin level is inversely and independently associated with the magnitude of weight loss induced by metformin.

Although a few studies have shown that there is no difference of weight change between metformin and placebo treatment in type 2 diabetes, in agreement with our study, the majority of studies have shown a moderate weight loss after metformin treatment. ${ }^{9-17}$ The discrepancy may be ascribed to different ethnicities and characteristics of subjects enrolled in the different studies. The magnitude of weight loss varies slightly among the studies. Our study showed that 16-week metformin monotherapy caused a weight loss of $2.4 \mathrm{~kg}$ in Chinese patients with newly-diagnosed type 2 diabetes. Similarity, the Diabetes Prevention Program (DPP) study showed that, within the first 3 years, metformin treatment induced weight loss of approximately $2.9 \mathrm{~kg}$. This weight loss effect persisted up to 8 years. ${ }^{17}$ Another study performed in Chinese patients showed that metformin lead to approximately $1.88 \mathrm{~kg}$ and $1.89 \mathrm{~kg}$ of weight loss after 24-week and 48-week treatment, respectively. ${ }^{18}$

Although metformin could induce a moderate weight loss, as our and most other studies have demonstrated, the International Diabetes Federation, American Diabetes Association and European Association for the Study
Table 2. Weight loss determinants by correlation coefficient

\begin{tabular}{|c|c|c|}
\hline & $r$ & $p$-value \\
\hline Age & 0.009 & 0.873 \\
\hline Weight & 0.257 & 0.000 \\
\hline BMI & 0.135 & 0.020 \\
\hline Waistline & 0.156 & 0.007 \\
\hline Serum albumin & -0.207 & 0.000 \\
\hline Hypertension & -0.024 & 0.682 \\
\hline GHBA1C & -0.043 & 0.467 \\
\hline FBG & 0.068 & 0.249 \\
\hline UA & -0.006 & 0.918 \\
\hline Cumulative dosages & 0.047 & 0.423 \\
\hline LDL-C & -0.041 & 0.486 \\
\hline TG & 0.088 & 0.134 \\
\hline CRP & 0.077 & 0.527 \\
\hline $\mathrm{HDL}$ & 0.104 & 0.077 \\
\hline TCHOL & -0.020 & 0.730 \\
\hline
\end{tabular}

Table 3. Possible predictors of weight loss after 16-week metformin therapy

\begin{tabular}{|l|c|c|}
\hline \multicolumn{1}{|c|}{ Variable entered } & $\begin{array}{c}\text { Standardized } \\
\text { coefficients }\end{array}$ & p-value \\
\hline Intercept & -2.89 & 0.0149 \\
\hline BMl & 0.22 & \\
\hline
\end{tabular}

of Diabetes recommend metformin as the first-line treatment in all newly-diagnosed patients, regardless of weight. ${ }^{12-17,19}$ However, few studies have assessed metformin in Asian countries, including China. Because of the differences of ethnicity and lifestyle, Asian populations have lower BMIs and lower rates of obesity compared to populations in Western countries. ${ }^{20}$ The cut-off point of "normal weight" from overweight for Asians $\left(B M I \leq 23.9 \mathrm{~kg} / \mathrm{m}^{2}\right)$ is lower than that for Caucasians $\left(B M I \leq 24.9 \mathrm{~kg} / \mathrm{m}^{2}\right) .{ }^{21,22}$ Only half of the patients with newly-diagnosed type 2 diabetes have followed the guidelines in China. ${ }^{7}$ The low compliances of both prescribing doctors and patients may be mainly ascribed to a lack of 
strong evidence on the effects of metformin on weight in Chinese patients with normal weight. The present analysis has addressed this issue. Our results showed that patients who were heavier at baseline achieved more weight loss after 16-week metformin treatment. Moreover, there is a linear correlation between weight at baseline and weight loss after 16-week metformin treatment. After 16-week metformin treatment, the proportion of obese subjects decreased, whereas the proportion of subjects with normal weight increased slightly. The most modest weight loss or unchanged weight (weight loss $\leq 0.5 \mathrm{~kg}$ ) happened among the patients with lowest weight and BMI at baseline. Therefore, as almost all international guidelines recommend, Asian patients with relatively lower BMIs may be treated with metformin without worrying about excessive weight loss.

The most common adverse events of metformin are mild to moderate gastrointestinal symptoms, which are well documented. ${ }^{4}$ However, the effects of gastrointestinal AE on weight are still unknown. Uncomfortable gastrointestinal symptoms, including vomiting, nausea and diarrhea, may reduce food intake for a certain period until the symptoms become tolerable, in which case, a higher rate of gastrointestinal AE may cause more weight loss. On the other hand, these gastrointestinal symptoms may also lead to lower compliance and affect the absorption efficiency of metformin in the epithelium of the intestine for a long time, which would lead to less weight loss. Therefore, the effects of gastrointestinal AE on weight depend on combinational actions. Our analysis shows that the weight loss induced by metformin is not associated with its gastrointestinal AE.

Consistent with another study, we did not find a correlation between glucose control and weight reduction. ${ }^{18}$ In addition, weight loss did not correlate with the accumulated administration dosages of metformin. Therefore, it is possible that metformin can be developed as an anti-obesity agent independent of its hypoglycemic effect. Further studies are required to demonstrate the possible efficiency and safety of metformin as a credible weight loss drug in obesity, independent of treating diabetes.

Although weight loss is often reported in patients with type 2 diabetes treated with metformin, the mechanisms of metformin-induced weight loss are still unclear. A recent work has suggested that metformin reduces weight through affecting appetite signals in the brain, fat oxidation and fat storage in the liver. ${ }^{23}$ Understanding the metformin-induced weight loss mechanisms may lead to developing optimal treatment strategies for preventing type 2 diabetes and obesity.

The limitation of our study is that there was no placebo-controlled group, for ethical reasons. Therefore, we could not exclude the effect of lifestyle change on weight, since all participants were advised to be on a diet and exercise regularly. However, the AHEAD study has proved that lifestyle modification only is difficult to prevent the weight of obesity from rebounding. ${ }^{24}$ Another limitation is that we follow the patients with newly-diagnosed type 2 diabetes for only 16 weeks. Long term investigation of the predictive factors of weight loss should be acquired.

\section{References}

1. Currie CJ, Poole CD, Gale EA. The influence of glucose-lowering therapies on cancer risk in type 2 diabetes. Diabetologia. 2009;52:1766-1777.

2. Mahmood K, Naeem M, Rahimnajjad NA. Metformin: The hidden chronicles of a magic drug. Eur J Intern Med. 2013;24:20-26.

3. Effect of intensive blood-glucose control with metformin on complications in overweight patients with type 2 diabetes (UKPDS 34). UK Prospective Diabetes Study (UKPDS) Group. Lancet. 1998;352:854-865.

4. Scarpello JH, Howlett HC. Metformin therapy and clinical uses. Diabetes \& vascular disease research. Official Journal of the International Society of Diabetes and Vascular Disease. 2008;5:157-167.

5. Inzucchi SE, Bergenstal RM, Buse JB, et al. Management of hyperglycaemia in type 2 diabetes, 2015: A patient-centred approach. Update to a Position Statement of the American Diabetes Association and the European Association for the Study of Diabetes. Diabetologia. 2015;58:429-442.

6. Ji L, Li H, Guo X, Li Y, Hu R, Zhu Z. Impact of baseline BMI on glycemic control and weight change with metformin monotherapy in Chinese type 2 diabetes patients: phase IV open-label trial. PloS One. 2013;8:e57222.

7. Ji L, Newman J, Lu J, Cai X. Understanding the standard of care in the treatment of type 2 diabetes in China: Results from a national survey. Chinese Medical Journal. 2014;27:3524-3529.

8. Meraviglia P, Angeli E, Del Sorbo F, et al. Risk factors for indinavir-related renal colic in HIV patients: predictive value of indinavir dose/body mass index. Aids. 2002;16:2089-2093.

9. Mather KJ, Verma S, Anderson TJ. Improved endothelial function with metformin in type 2 diabetes mellitus. Journal of the American College of Cardiology. 2001;37:1344-1350.

10. Saenz A, Fernandez-Esteban I, Mataix A, Ausejo M, Roque M, Moher D. Metformin monotherapy for type 2 diabetes mellitus. The Cochrane Database of Systematic Reviews. 2005,CD002966.

11. Ito $H$, Ishida $H$, Takeuchi $Y$, Antoku S, Abe M, Mifune M, Togane M. Long-term effect of metformin on blood glucose control in nonobese patients with type 2 diabetes mellitus. Nutrition \& Metabolism. 2010;7:83.

12. Stumvoll M, Nurjhan N, Perriello G, Dailey G, Gerich JE. Metabolic effects of metformin in non-insulin-dependent diabetes mellitus. The New England Journal of Medicine. 1995;33:550-554.

13. Ong CR, Molyneaux LM, Constantino MI, Twigg SM, Yue DK. Longterm efficacy of metformin therapy in nonobese individuals with type 2 diabetes. Diabetes Care. 2006;29:2361-2364.

14. Fontbonne A, Diouf I, Baccara-Dinet M, Eschwege E, Charles MA. Effects of 1-year treatment with metformin on metabolic and cardiovascular risk factors in non-diabetic upper-body obese subjects with mild glucose anomalies: A post-hoc analysis of the BIGPRO1 trial. Diabetes \& Metabolism. 2009;35:385-391.

15. Seifarth C, Schehler B, Schneider HJ. Effectiveness of metformin on weight loss in non-diabetic individuals with obesity. Experimental and clinical endocrinology \& diabetes: Official journal, German Society of Endocrinology [and] German Diabetes Association. 2013;121:27-31.

16. Malin SK, Nightingale J, Choi SE, Chipkin SR, Braun B. Metformin modifies the exercise training effects on risk factors for cardiovascular disease in impaired glucose tolerant adults. Obesity. 2013;21:93-100.

17. Diabetes Prevention Program Research G. Long-term safety, tolerability, and weight loss associated with metformin in the Diabetes Prevention Program Outcomes Study. Diabetes Care. 2012; 35:731-737.

18. Yang W, Liu J, Shan Z, et al. Acarbose compared with metformin as initial therapy in patients with newly diagnosed type 2 diabetes: an open-label, non-inferiority randomised trial. The Lancet Diabetes \& Endocrinology. 2014;2:46-55. 
19. Inzucchi SE, Bergenstal RM, Buse JB, et al. Management of hyperglycaemia in type 2 diabetes: A patient-centered approach. Position statement of the American Diabetes Association (ADA) and the European Association for the Study of Diabetes (EASD). Diabetologia. 2012;55:1577-1596.

20. Socolovsky M, Paez MD. A literature review of intercostal-to-musculocutaneous-nerve transfers in brachial plexus injury patients: Does body mass index influence results in Eastern versus Western countries? Surgical Neurology International. 2013;4:152.

21. Obesity: Preventing and managing the global epidemic. Report of a WHO consultation. World Health Organization technical report series 2000;894;i-xii:1-253.

22. Zhou BF, Cooperative Meta-Analysis Group of the Working Group on Obesity in C. Predictive values of body mass index and waist circumference for risk factors of certain related diseases in Chinese adults--study on optimal cut-off points of body mass index and waist circumference in Chinese adults. Biomedical and environmental sciences. BES. 2002;15:83-96.

23. Malin SK, Kashyap SR. Effects of metformin on weight loss: Potential mechanisms. Current Opinion in Endocrinology, Diabetes, and Obesity. 2014;21:323-329.

24. Look ARG, Wadden TA, West DS, et al. The Look AHEAD study: A description of the lifestyle intervention and the evidence supporting it. Obesity. 2006;14:737-752. 